\title{
Pé diabético: estratégias para prevenção
}

\author{
Kattia Ochoa-Vigo ${ }^{1}$ \\ Ana Emilia Pace ${ }^{2}$
}

Ochoa-Vigo K, Pace AE. Pé diabético: estratégias para prevenção. Acta Paul Enferm 2005; 18(1):100-9.

\begin{abstract}
RESUMO: Aproximadamente 50\% das amputações não-traumáticas em membros inferiores ocorrem entre pessoas com diabetes. As amputações são precedidas por úlceras, caracterizadas por lesões cutâneas, com perda do epitélio, que podem estender-se até a derme ou chegar a tecidos profundos. As úlceras, geralmente, são acompanhadas de insensibilidade por neuropatia periférica crônica e associadas a pequenos traumas que se originam do uso de calçados inapropriados, dermatoses comuns ou manipulações incorretas dos pés. O objetivo deste estudo é descrever as principais estratégias para avaliar fatores de risco que conduzem ao desenvolvimento de complicações nos membros inferiores entre pessoas com diabetes, bem como considerá-las uma responsabilidade da equipe de assistência primária. É um trabalho de revisão da literatura, com ênfase na avaliação dos pés como medida preventiva fundamental, para identificar, precocemente, as alterações neurológica e vascular periféricas e disfunções biomecânicas. Destaca-se a avaliação dos calçados como fator externo.
\end{abstract}

Descritores: Diabetes mellitus; Fatores de risco; Pé diabético

- Artigo recebido em 06/04/04 e aprovado em 13/08/04

\section{INTRODUÇÃO}

Denomina-se pé diabético um estado fisiopatológico multifacetado, caracterizado por lesões que surgem nos pés da pessoa com diabetes e ocorrem como conseqüência de neuropatia em $90 \%$ dos casos, de doença vascular periférica e de deformidades ${ }^{(1)}$. As lesões geralmente decorrem de trauma e freqüentemente se complicam com gangrena e infecção, ocasionadas por falhas no processo de cicatrização ${ }^{(2-4)}$ as quais podem resultar em amputação, quando não se institui tratamento precoce e adequado ${ }^{(1)}$.
Cinqüenta por cento das amputações não-traumáticas de membros inferiores são atribuídas ao diabetes ${ }^{(2,5-6)}$ e o risco de amputação é 15 vezes maior do que na população geral ${ }^{(7)}$. Três anos após amputação de um membro inferior, a porcentagem de sobrevida do indivíduo é de $50 \%$, enquanto, no prazo de cinco anos, a taxa de mortalidade permanece de $39 \%$ a $68 \%{ }^{(8)}$. Estudo com pessoas diabéticas hospitalizadas, recentemente publicado, apresentou resultados semelhantes a esses ${ }^{(9)}$.

Dados da América do Norte indicam que de $9 \%$ a $20 \%$ das pesso- as com diabetes precisaram ser submetidas a uma segunda amputação, após doze meses da primeira, e que, nos cinco anos seguintes à amputação inicial, de $28 \%$ a $51 \%$ dos que sobreviveram precisarão submeterse a uma segunda intervenção no mesmo membro ${ }^{(8)}$. Outro estudo, entretanto, reportou um reduzido percentual de novas e maiores amputações no mesmo membro ou no contralateral, resultados que podem ser atribuídos ao processo educativo instituído aos participantes, abordando cuidados com os pés, bem como fornecimento de órtese aos mesmos ${ }^{(9)}$.

\footnotetext{
1 Doutoranda do Programa Interunidades da Escola de Enfermagem e Escola de Enfermagem de Ribeirão Preto, Universidade de São Paulo. Bolsista da Fundação de Amparo à Pesquisa do Estado de São Paulo - FAPESP. E-mail: kovpe@latinmail.com

2 Professora Doutora da Escola de Enfermagem de Ribeirão Preto, Universidade de São Paulo. E-mail: aepace@eerp.usp.br
} 
Estudo realizado no município do Rio de Janeiro, mediante a técnica de captura-recaptura, quando comparada à vigilância passiva, encontrou incidência sete vezes maior na população diabética entre 55 e 74 anos, do que na população geral. A estimativa anual de amputações maiores de membros inferiores na população geral foi de 31,3/100.000 habitantes, porém, quando relacionada ao diabetes, a cifra elevou-se para 209/100.000 ${ }^{(10)}$.

Freqüentemente, as amputações nas pessoas com diabetes são precedidas de úlceras $^{(1,3,5,11-12)}$, caracterizadas por lesões cutâneas com perda do epitélio, as quais se estendem até a derme ou a atravessam e chegam aos tecidos mais profundos ${ }^{(6,13)}$, envolvendo algumas vezes ossos e músculos ${ }^{(14)}$.

As úlceras em pessoas com diabetes são responsáveis por grande percentual de morbimortalidade e hospitalização ${ }^{(4)}$ e têm um período de internação 59\% mais prolongado que as pessoas com diabetes sem processos ulcerativos $^{(6)}$. O gasto com as úlceras na fase prodrômica aumenta de 1,5 a 2,4 vezes, se comparado ao dispendido com pessoas sem úlceras, e, quando o problema se instala, este custo eleva-se a 5,4 vezes ${ }^{(15)}$, duplicando o índice de mortalidade dessas pessoas ${ }^{(16)}$.

As hospitalizações causadas por pé diabético geralmente são prolongadas e recorrentes, exigindo grande número de consultas ambulatoriais e necessidade de cuidado domiciliar $^{(15,17)}$. O processo de cicatrização das lesões ocorre de seis a 14 semanas, requerendo um período de hospitalização de 30-40 dias, em países desenvolvidos, enquanto, no Brasil, essa média fica em torno de 90 dias $^{(1)}$.

Observa-se que $85 \%$ dos casos graves que necessitam de hospitalização são causados por úlceras superficiais ou lesões pré-ulcerativas, as quais apresentam diminuição da sensibilidade devido à neuropatia di- abética. Esses casos estão associados a pequenos traumas originados por uso de calçados impróprios, dermatoses comuns, manipulações incorretas dos pés ou unhas, seja pela própria pessoa ou por outras não habilitadas ${ }^{(11)}$.

As lesões do pé diabético resultam da combinação de dois ou mais fatores de risco que atuam concomitantemente e podem ser desencadeadas, tanto por traumas intrínsecos como extrínsecos, associados à neuropatia periférica, à doença vascular periférica e à alteração biomecânica ${ }^{(1)}$.

A neuropatia periférica constitui-se no fator significante nesses casos, conduzindo à lesão/ulceração do membro inferior. Encontra-se presente em aproximadamente $80 \%$ a $85 \%$ dos casos ${ }^{(1,18)}$ e pode comprometer as fibras sensitivas, as motoras e as autonômicas. O componente sensitivo produz perda gradual da sensibilidade à dor, percepção da pressão plantar, temperatura e propriocepção. Quanto ao componente motor, este contribui para atrofia e fraqueza dos pequenos músculos dorsais, desencadeando desequilíbrio nos tendões flexores e extensores, deformidades e alterações no modo de caminhar. Já o componente autonômico reduz ou suprime o suor nos pés, deixandoos secos e predispondo-os a rachaduras e fissuras, além de desencadear alterações arteriovenosas ${ }^{(1,13,18)}$. No que diz respeito às mudanças neuropáticas em geral, estas afetam primeiramente os pés e, posteriormente, progridem em direção à perna ${ }^{(19)}$.

O mecanismo da neuropatia diabética não está claro, porém é atribuído à deterioração da função do nervo, subjacente às anormalidades metabólicas e isquemia endoneural da circulação microvascular. Quando a neuropatia periférica se instala, é irreversível, portanto, é particularmente importante que pessoas com diagnóstico recente tenham precau- ção em relação à sua progressão, mantendo adequado controle glicêmico ${ }^{(19)}$.

No que se refere à doença vascular periférica, esta representa uma das principais causas de comprometimento das úlceras nos pés das pessoas com diabetes, devido à aterosclerose das artérias periféricas. Esta doença é quatro vezes mais provável de ocorrer em pessoas com diabetes do que na população em geral e sua incidência aumenta gradualmente com a idade e com a duração da doença ${ }^{(20)}$. Constitui-se em importante fator de risco para ulceração e amputação, devido ao comprometimento da perfusão sangüínea em membros inferiores, pois priva os tecidos de adequado fornecimento de oxigênio, nutrientes e antibióticos que prejudica a cicatrização das úlceras, podendo conseqüentemente levar à gangrena ${ }^{(4)}$.

Em pessoas com diabetes, é comum o aparecimento precoce do processo aterosclerótico da doença vascular periférica, o qual pode progredir mais rapidamente do que na população geral ${ }^{(20)}$. Este processo aterosclerótico acelerado produz hipercoagulação e aumento da resistência vascular ${ }^{(20-21)}$, precipitando a obstrução das artérias e arteríolas distais que, conseqüentemente, dificulta o fluxo sangüíneo. Inicialmente são obliteradas as pequenas artérias tibial e peroneal, na altura do joelho e do tornozelo, que podem ser agravadas pelo tabagismo e dislipidemia ${ }^{(20)}$.

Após trauma, pode surgir uma úlcera dolorosa essencialmente isquêmica ou neuroisquêmica, às vezes indolor, quando associada à insensibilidade. Os dedos podem tornar-se isquêmicos ou necrosados diante de trombose séptica, traumas, edemas leves ou infecções por comprometimento de arteríolas semiocluídas ${ }^{(1,13,18)}$. Podem-se identificar, nessa situação ainda, pulsos nas artérias dorsais do pé ou tibial posterior, mesmo em presença de isquemia 
nos dedos, pois nem sempre a doença dos grandes e pequenos vasos progride na mesma velocidade ${ }^{(20)}$.

Em conseqüência da perda da sensibilidade protetora devido à polineuropatia simétrica distal associada ao diabetes, podem associarse a este quadro fatores de risco relacionados à biomecânica da marcha, causando/agravando lesões nos pés pelo trauma repetitivo.

Entre as alterações biomecânicas, estão as relacionadas com o movimento do corpo, incluindo as forças verticais, horizontais e de acomodação. Na mensuração da biomecânica corporal, destacam-se a força-reação do solo e a distribuição da pressão plantar que ocorreu durante a fase de apoio ${ }^{(22)}$. Qualquer limitação, na extensão do movimento das articulações do pé, interrompe a mecânica da marcha, o que leva a pessoa a desenvolver um passo disfuncional o qual, certamente, produzirá um dano estrutural maior no pé ${ }^{(13)}$.

Nas pessoas com neuropatia periférica, as deformidades ósseas desenvolvidas nas cabeças metatarsianas dos pés e no antepé representam áreas de excessiva pressão durante a fase de propulsão do calcâneo e de apoio plantar no ciclo da marcha ${ }^{(13)}$. Isso porque, quando o calcâneo se eleva do chão, transfere-se o peso do corpo para o antepé e dedos. Assim, a força de pressão gerada depende da velocidade na marcha, ou seja, quanto maior a velocidade, maiores serão a força de reação e a pressão.

Normalmente, a região plantar é protegida por um coxim adiposo, o qual dissipa as forças do peso para todas as direções. Portanto, deslocamentos ou atrofias nesses coxins provocarão aumento da pressão debaixo deles, principalmente sob as cabeças metatarsianas com deformidade rígida, elevando a pressão nessa região ${ }^{(13)}$. Dessa forma, se a magnitude de forças for suficientemente elevada em uma região plan- tar, a ocorrência de qualquer perda de pele ou hipertrofia do estrato córneo (calos) aumentará o risco de ulceração por duas ordens de magnitude ${ }^{(13)}$.

Entende-se que o risco de ulceração é proporcional ao número de fatores de risco ${ }^{(23)}$, e que estes aumentam 1,7 vez em pessoas com diagnóstico de neuropatia periférica, subindo para 12 vezes em pessoas com neuropatia e deformidade do pé e para 36, naquelas com neuropatia, deformidade e amputação prévia, quando comparadas a pessoas sem fatores de risco ${ }^{(13)}$.

Com base nas múltiplas causas que favorecem o desencadeamento de lesões e ulcerações nos pés de pessoas com diabetes e reconhecendo a vulnerabilidade das mesmas para amputações de membros inferiores, é que se reforça a necessidade de compreensão desse complexo processo pela equipe multiprofissional, para que ela se envolva com os mesmos.

Assim, ao se avaliar uma pessoa com diabetes, enfatizando-se a prevenção das complicações nos pés, o profissional deve buscar não só a influência dos fatores que poderão estar envolvidos direta ou indiretamente na instalação dessas complicações, mas também as conseqüências destes na vida da pessoa, destacando-se, principalmente, o controle do diabetes. No atendimento a essa clientela, o profissional deve incluir um exame minucioso dos pés, levando em consideração as características da pele e fâneros ${ }^{(24)}$, segundo as propostas apresentadas na literatura e os recursos disponíveis.

Com base no exposto, este estudo tem como objetivo descrever as principais estratégias utilizadas na avaliação dos membros inferiores das pessoas com diabetes, destacando os achados que indicam a presença dos fatores de risco que levam a complicações em pés, a fim de integrar essas estratégias nos programas implementados pela equipe de assistência primária direcionados a essa clientela.

Trata-se de uma revisão da literatura nacional e internacional, com inclusão de estudos descritivos e experimentais relevantes, localizados em bases eletrônicas de dados e referências citadas. Consideraram-se estudos que poderiam subsidiar a prática profissional, ou seja, incluir estratégias e ações que identificassem, precocemente, as pessoas em condições de risco, visando a contribuir para a melhoria do prognóstico da doença e qualidade de vida.

\section{AVALIAÇÃO DOS PÉS E PREVENÇÃO DE COMPLICAÇÕES}

Estudos vêm ressaltando a necessidade de os profissionais de saúde avaliarem os pés das pessoas com diabetes de forma minuciosa e com freqüência regular, bem como desenvolverem atividades educativas, visando a melhorar o autocuidado, principalmente a manutenção de um bom controle glicêmico ${ }^{(1,2,4,19,21,25-31)}$.

A avaliação dos pés constitui-se em passo fundamental na identificação dos fatores de risco que podem ser modificados, o que, conseqüentemente, reduzirá o risco de ulceração e amputação de membros inferiores nas pessoas com diabetes ${ }^{(30)}$.

Autores destacam que a diminuição da sensação protetora, caracterizada pela redução da sensação dolorosa de trauma na pele ${ }^{(3,32-37)}$, bem como a ausência parcial ou total do reflexo Aquileu ${ }^{(14,26,38-39)}$ constituem sinais precoces de futuros processos ulcerativos nos pés, significando alto risco para o desenvolvimento de complicações. Ambas as alterações podem ser avaliadas mediante testes que utilizam vários tipos de instrumentos, desde os mais sofisticados aos mais simples. Um deles é o monofilamento SemmesWeistein (SW) 5.07 e outro o biotesiômetro (VPT) que associados constituem os melhores preditores 
de processos ulcerativos em pessoas com diabetes, atingindo uma sensibilidade de $100 \%$ e uma especificidade de $77 \%{ }^{(40)}$.

O monofilamento SW é um instrumento manual que contém uma fibra de náilon com força de 10 gramas, sendo aplicado à sola do pé, a um ângulo de 90 graus ${ }^{(36)}$, mediante a técnica da resposta sim-não ao toque do aparelho em 10 regiões do pé (primeiro, terceiro e quinto dígitos plantares; primeira, terceira e quinta cabeça dos metatarsos plantares; laterais esquerda e direita do meio plantar; calcâneo e dorso entre primeiro e segundo dedos). A técnica instrui a pessoa a dizer sim, a cada vez que perceber a aplicação da fibra. A inabilidade, para distinguir o monofilamento SW em quatro pontos ou mais, é indicativa de perda da sensação de proteção ${ }^{(37,40-41)}$.

O VPT é um dispositivo manual que contém um sensor que vibra a $100 \mathrm{~Hz}$ ao entrar em contato com a pele, conectado a uma unidade-base por meio de um cordão elétrico. Esta unidade possui uma escala linear expressa através de voltagens que oscilam entre 0 (zero) e 50 (cinqüenta) volts. O dispositivo, aplicado à polpa do hálux, começa a se balancear verticalmente e a voltagem vai aumentando na unidade-base até que a pessoa seja capaz de perceber a vibração ${ }^{(40)}$. O padrão de vibração considerado normal é menor que 25 volts e as voltagens com vibrações mais intensas ou iguais indicam comprometimento neuropático ${ }^{(35,37,40,42)}$.

O custo estimado do VPT é elevado, em comparação ao monofilamento SW, girando em torno de U\$ 400,00 (quatrocentos dólares) no mercado internacional, ao passo que o monofilamento SW pode ser encontrado no mercado nacional por $\mathrm{R} \$ 7,40$ (sete reais e quarenta centavos). Por essa razão, o monofilamento SW é o instrumento portátil mais utilizado para identificar o comprometimento da sensibilidade protetora plantar, como sinal indi- cativo de neuropatia periférica, seja pelo seu custo-efetividade ou pela maior reprodutibilidade demonstrada em estudos prospectivos ${ }^{(43)}$.

Cabe destacar que o monofilamento SW perde sua calibração após uso repetitivo e, em conseqüência disso, pode subestimar a incidência da perda da sensação protetora. Para controlar essa limitação, recomenda-se deixá-lo em restauração durante 24 horas, após aplicação em 10 pessoas ${ }^{(21)}$. Na prática, é possível observar-se diminuição da força do monofilamento SW em menor número de aplicações, fato que pode estar relacionado à influência do clima tropical, à qualidade do produto e à técnica utilizada. Por tais motivos, os profissionais devem tomar providências que garantam a utilização segura do aparelho para uma avaliação efetiva.

As pessoas da terceira idade apresentam (pelo próprio processo do envelhecimento) diminuição da sensibilidade protetora, o que não está relacionado, necessariamente, com a neuropatia periférica. Por isso, ao fazer a avaliação dessa clientela, o profissional deve considerar as características da pele à procura de sinais de desidratação, fissuras e diminuição da transpiração em membros inferiores (27), bem como de descamação.

Mediante a importância da avaliação clínica da sensibilidade vibratória, alguns autores ${ }^{(14,44)}$ recomendam utilizar o diapasão de 128 $\mathrm{Hz}$, instrumento manual semiquantitativo que deve ser aplicado, de forma perpendicular, sobre a parte óssea dorsal da falange distal do hálux. Frente à incapacidade da percepção de vibração, o teste deve ser repetido em segmentos mais próximos, como o maléolo ou tuberosidade da tíbia. O teste é qualificado como positivo (sensibilidade vibratória preservada) quando, após três aplicações, a pessoa responde, corretamente, a pelo menos duas indagações ${ }^{(44)}$.
Quanto ao reflexo Aquileu, este pode ser avaliado ao se colocar a pessoa ajoelhada sobre a margem de uma cadeira com respaldo, e o examinador por trás posicionará o pé em suave dorsiflexão, de modo a causar estiramento muscular, e percutirá no tendão ${ }^{(45)}$.

Outro aspecto a se considerar na avaliação é a alteração biomecânica ou osteoarticular dos pés ${ }^{(30,32,46)}$. Essa alteração poderá produzir deformidades estruturais (hálux valgo, pododáctilos em garra, pé plano, pé cavo), expondo os pés a uma distribuição anormal da pressão plantar em regiões, às vezes, não protegidas anatomicamente. Esta constante pressão contribui para o desenvolvimento de calosidades nas pessoas com diabetes ${ }^{(41)} \mathrm{e}$, paralelamente, pode atuar como feedback positivo, no sentido de acentuar/agravar as calosidades ${ }^{(46)}$.

Altas pressões em pontos ósseos na região plantar ${ }^{(23,35,42,47-48)}$, associadas a calosidades ${ }^{(41,49)}$, são grandes preditores de processos ulcerativos, por isso, é necessário determinar os locais específicos da hiperpressão, para se implementarem atividades de prevenção e controle.

Vários modelos de sistemas comercialmente disponíveis vêm sendo utilizados para esse fim (sistema de impressão de Harris-Beath, Pedobarografia, sistema F-scan, sistema EMED-SF, Mikro EMED, Pedar, sistema Paromed, sistema Ormes, sistema COSINOS EDV, sistema Pedcad, sistema HALM, sistema Scram-Mel-Card, Planscan platform, dentre outros), considerando diferentes tipos de sensores que quantificam a pressão plan$\operatorname{tar}^{(14,23)}$. Métodos semiquantitativos também são propostos, como uma alternativa mais simples para o diaa-dia, e dentre eles destaca-se o Podotrack footprint system que quantifica a pressão plantar por meio da observação da intensidade da impressão cinzenta em uma papeleta ${ }^{(14)}$. Porém, o mecanismo para 
medir a pressão plantar ainda não está bem definido, havendo necessidade de maiores pesquisas a respeito ${ }^{(46-47)}$. Também, um exame clínico que inclua avaliação de calosidades na região plantar, por meio de simples inspeção e palpação, pode ser uma outra prática alternativa.

Autores identificaram que a média da pressão plantar entre os diferentes pontos avaliados foi mais elevada em pessoas diabéticas com problemas neuroisquêmicos, quando comparadas àquelas somente com neuropatia. No estudo, destacou-se que os primeiros possuem calosidades em menor quantidade, fato que pode estar relacionado à baixa supressão de sangue nos membros inferiores, o que levou os autores a concluir que o aumento da pressão plantar em pessoas diabéticas com problemas isquêmicos, incluídos os neuroisquêmicos, nem sempre está associado a futuros processos ulcerativos na região plantar, demonstrando que existem diferentes mecanismos para formação das úlceras, tanto em pés neuroisquêmicos, como nos neuropáticos ${ }^{(50)}$.

A elevação da pressão plantar também está associada à limitação da mobilidade articular ${ }^{(23,35,46,51-52,)}$, destacando-se a metatarsofalangiano, subtalar e o tornozelo. O movimento da articulação subtalar é de especial interesse quando se trata de pés de pessoas com diabetes, pois qualquer redução na sua mobilidade poderá causar aumento da pressão plantar durante a marcha ${ }^{(52)}$, especialmente ao se considerar que sua função é absorver o impacto do calcâneo no chão, no momento do choque ${ }^{(53)}$.

Durante o processo de marcha, espera-se que, primeiramente, o pé adapte seu movimento a superfícies irregulares e acidentadas e, segundo, que proporcione rigidez à fase da propulsão. Nessas fases (de choque e de propulsão), a articulação subtalar executa os movimentos de pronação e de supinação e, em pre- sença de alguma limitação articular, o pé será incapaz de realizar o mecanismo de absorção, diminuindo sua habilidade para manter uma pressão normal ${ }^{(52)}$. Porém, se a essa condição associarem-se a postura e os movimentos instáveis, presentes em pessoas com diabetes ${ }^{(14,54)}$, o risco de sofrer qualquer prejuízo durante a marcha poderá elevar-se.

O fator essencial, entre limitação da mobilidade articular do pé e deformidade que levam à ulceração plantar, parece estar associado à pressão plantar elevada e à neuropatia periférica. Altas pressões ocorrem em regiões com deformidades e nesses locais, com freqüência, aparece uma úlcera. Certamente, altas pressões plantares, de forma isolada, não causam ulceração, pois para sua ocorrência é necessária a co-existência de neuropatia periférica. Dessa forma, a limitação da mobilidade articular constitui-se no maior fator de alta pressão plantar e pode contribuir para o desencadeamento de ulceração em pés susceptíveis de neuropatia, nos quais a insensibilidade periférica e a microangiopatia, quando associadas, representam fatores predisponentes à ulceração $^{(52)}$.

Quanto à articulação metatarsofalangiana, esta pode ser avaliada por meio da dorsoflexão do hálux , mensurada com goniômetro simples, em uma amplitude de 50 graus. Reduções extremas da dorsoflexão do hálux em pessoas com diabetes e neuropatia elevam a pressão sob o dedo e podem desencadear úlceras $^{(46)}$. Para a avaliação da articulação subtalar, utiliza-se goniômetro especializado $^{(52)}$.

Na avaliação vascular periférica, o profissional deve interrogar a pessoa com diabetes sobre a presença de claudicação intermitente, dor ao repouso ou durante a noite ${ }^{(31)}$. A dor de claudicação aparece durante a marcha e cessa assim que esta termina. Sua gravidade depende da distância percorrida e da região afeta- da, tendo pior prognóstico quando aparece em caminhadas curtas e em regiões mais distais aos pés. Além desses, outros sinais isquêmicos podem estar presentes, tais como rubor de declive, palidez à elevação da perna, espessamento de unhas, pele atrofiada, fria e reluzente e diminuição de pêlos no dorso das extremidades ${ }^{(31)}$.

Na palpação de pulsos, o profissional deve considerar o tibial posterior e o pedioso que podem ser classificados em palpáveis ou não. Caso se identifique pulso diminuído ou ausente, é aconselhada uma segunda avaliação por um outro profissional e, ao se confirmarem ausência de pulsos e presença de outros sinais de comprometimento vascular, deve-se encaminhar a pessoa ao especialista.

Antecedentes de úlcera e amputação são informações importantes e sinais de alto risco em pessoas com diabetes $^{(1,2,21,25)}$. Nesses casos, o profissional deve adotar todas as intervenções de prevenção possíveis, a fim de garantir um adequado cuidado dos problemas apresentados pelas pessoas com diabetes. Dentre essas intervenções, salienta-se o uso constante de calçados apropriados de acordo com a necessidade de cada pessoa. Estudos apontam que os pontos de alta pressão, calosidades, deformidades nos pés, amputação de dedos, ou mesmo transmetatarsianos, são problemas que podem ser corrigidos com calçados confortáveis ou confeccionados sob medida, coadjuvados com palmilhas ${ }^{(9,20-21,25,30,34,37,46)}$.

Estudo prospectivo, realizado em hospital de ensino para avaliar pessoas com diabetes de alto risco, identificou menor recorrência de ulcerações no grupo que havia recebido calçados especialmente confeccionados ${ }^{(55)}$. Um outro estudo mostrou, também, menor recorrência de úlceras e amputações entre aqueles que usaram calçados terapêuticos e receberam intenso treinamento 
educativo ${ }^{(9)}$. Em contrapartida, estudo randomizado, recentemente publicado $^{(56)}$, não apresentou diferença significativa quanto à recorrência de úlceras em pessoas que utilizaram calçados terapêuticos, comparando dois tipos de solados e aqueles que continuaram a utilizar calçados convencionais. O estudo ainda destacou que calçados especiais podem ser benéficos aos pacientes que não têm assistência especializada de cuidado aos pés e àqueles com deformidades graves nessa mesma região.

Dessa forma, pessoas com diabetes devem ser aconselhadas a utilizar calçados apropriados que se ajustem à anatomia de seus pés. Quando identificada neuropatia, mesmo sem presença de deformidades estruturais, recomenda-se uso de palmilhas, para reduzir e amortecer o efeito de tensão repetitiva. Entretanto, indicam-se calçados especiais conforme a avaliação de peritos, quando identificadas deformida$\operatorname{des}^{(21,57)}$. Dentre as características dos calçados, destacam-se aqueles com profundidade extra, que permitem a adaptação de palmilhas removíveis; os acolchoados internamente; aqueles com ausência de costuras interiores e sistema de fechamento ajustável (velcro ou cadarço); os que têm ponta quadrada e saltos de até três centímetros. É importante que os calçados cubram totalmente os dedos e o calcanhar e que sejam confeccionados com couro macio ou lona.

Embasado na experiência, pesquisador ${ }^{(32)}$ indica características importantes a serem consideradas nos calçados terapêuticos, mediante o desenvolvimento de um algoritmo. Enfatiza que a palmilha, unicamente, influencia na distribuição da pressão, visto que a redução da fricção exigiria uma ótima fixação dos pés aos calçados.

Em relação às intervenções educativas de autocuidado, autores ressaltam as direcionadas ao exame

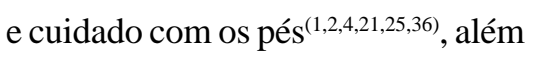
de enfatizarem bom controle glicêmico, da pressão sangüínea, colesterol (58), dieta e realização regular de exercícios.

A educação tem como objetivo sensibilizar, motivar e mudar atitudes da pessoa que deve incorporar a informação recebida, sobre os cuidados com os pés e calçados ${ }^{(1)}$, no seu dia-a-dia, reduzindo, conseqüentemente, o risco de ferimento, úlceras e infecção (31).

Revisão de estudos prospectivos sobre intervenções educativas bem estruturadas identificou melhoria relativa do conhecimento com cuidado dos pés, assim como mudança de conduta das pessoas com diabetes ${ }^{(29)}$. No entanto, ainda é difícil evidenciar o impacto da educação nessa população ${ }^{(43)}$. Acredita-se, porém, que a acuidade visual, obesidade, mobilidade limitada e problemas cognitivos devam interferir nas habilidades de autocuidado apropriado com os pés ${ }^{\left({ }^{30}\right)}$, mesmo não se considerando as condições socioeconômicas que, em suma, determinam o estilo e a qualidade de vida.

Alguns estudos prospectivos relataram resultados favoráveis e um deles mostrou significativa diminuição da recorrência de úlceras e poucas amputações nas pessoas do grupo experimental, os participantes receberam assistência de um podiatra e de um educador diabetologista, além de calçados especiais durante 24 meses. Os sujeitos eram avaliados a cada três meses no hospital, onde reforçavam-se as atividades educativas de acordo com as necessidades identificadas que constavam de: apresentação de vídeo e provisão de materiais ilustrativos ${ }^{(59)}$. Um outro estudo também mostrou redução efetiva de úlceras, após o desenvolvimento de um programa educativo que durou seis anos ${ }^{(60)}$. Nesse período, o percentual acumulado de úlceras no grupo de intervenção foi de $3,1 \%$ e no controle, de $31,6 \%$. Os participantes eram in- seridos em programas de pé, compondo grupos de até seis pessoas, durante uma semana. Na primeira sessão, os profissionais avaliaram, de forma individualizada, as características dos pés dos participantes, destacando a percepção sensorial, habilidades e limitações do auto-cuidado, com aconselhamento para consulta mensal com o podiatra.

Elementos básicos na educação incluem: inspeção diária de pés, meias e calçados; higiene dos pés com água morna e sabonete neutro, evitando deixá-los em imersão, com orientação de enxugá-los cuidadosamente; remoção de pequenas calosidades com lixa de papel ou pedrapomes; corte de unhas retas não muito rentes; uso de creme ou óleo hidratante; calçados apropriados que propiciem conforto aos dedos, com um mínimo de costuras internas, devendo o forro permitir a evaporação do suor. Não se devem utilizar produtos químicos para remoção de calos/verrugas, nem objetos cortantes ou pontiagudos ${ }^{(4,21,31,61-62)}$, devido ao perigo de provocarem ferimentos na pele.

Quando a pessoa com diabetes possui dificuldade visual ou outro tipo de limitação, uma outra deveria ser preparada para realizar tais cuidados, destacando-se a avaliação diária dos pés à procura de algum sinal de lesão.

Quanto ao controle glicêmico, estudos referem que um controle glicêmico pobre aumenta o risco de neuropatia e amputação nas pessoas com diabetes ${ }^{(7,63)}$, o que é comprovado por revisões recentes, que evidenciaram que um controle glicêmico eficaz reduz o risco de retinopatia, neuropatia e nefropatia no diabetes tipo $2^{(58,62,64)}$; assim, seu parâmetro de medida na hemoglobina glicada $\left(\mathrm{HbA}_{1 \mathrm{c}}\right)$ constitui-se em uma das pedras angulares que podem retardar o desencadeamento e/ou controlar as doenças associadas ao diabetes, com conseqüente melhora na qualidade de vida. 


\section{CONSIDERAÇÕES FINAIS}

A partir da revisão, observa-se que o manejo dos pés da pessoa com diabetes é complexo, pois exige uma estreita colaboração e responsabilidade tanto dos pacientes, como dos profissionais, para rastrear os problemas reais e potenciais, evitando, assim, o desenvolvimento de complicações.

No atendimento a essa clientela, a equipe deve ser constituída basicamente, por médico generalista, enfermeiro especialista ou educador e podiatra ou quiropodista, os quais devem ter rápido acesso ao ortodista, radiologista, cirurgião vascular e ortopedista. Estes profissionais devem gerenciar os cuidados direcionados à população com diabetes, garantir um fluxo contínuo nos diferentes níveis de atenção à saúde ${ }^{(2-4,29,46,59)}$, para que as intervenções tenham o resultado e a eficácia esperada, visando a alcançar a meta proposta pela Declaração de St. Vicent, qual seja a de reduzir em $50 \%$ as amputações.

Diante da inexistência de podiatra ou quiropodista no Brasil, os cuidados básicos das lesões podem ser efetuados, nos níveis primário e secundário, por médicos generalistas, enfermeiros e diabetologistas, previamente treinados ${ }^{(11)}$. Em recente estudo ${ }^{(65)}$, os acidentes com pedicuros ou os domiciliares, para retirada de calos plantares, bem como o tratamento incorreto de lesões neuroisquêmicas, sinais súbitos de isquemia periférica e insensibilidade nos pés, além de outros relacionados, foram apontados como precursores das amputações.

Este último estudo ${ }^{(65)}$ mostrou, também, que $60 \%$ das pessoas submetidas à amputação possuíam mau controle metabólico, não tinham acesso a informações sobre cuidados preventivos, não aderiram ao tratamento clínico e apresentavam dificuldades econômicas. Por outro lado, destaca que esses problemas são passíveis de soluções, mediante medidas e tecnologias apropriadas, principalmente por serem de baixa complexidade assistencial.

No entanto, sabe-se que a redução das complicações nos pés, que conduzem à amputação, não depende unicamente dos recursos hospitalares, mas sim, da disponibilidade de medidas preventivas efetivas sobre os cuidados com os pés, bem como da oferta de programas educativos a toda a comunidade ${ }^{(33)}$. Esses dados são relevantes ao se considerar que a maioria das pessoas com diabetes é tratada pelo clínico geral da rede pública da comunidade (unidades básicas e unidades distritais de saúde), onde o aprazamento das consultas médicas pode variar de três meses a um ano.

Dessa maneira, a equipe da assistência primária deve conscientizar-se das necessidades e riscos a que estão sujeitas as pessoas com diabetes, principalmente aquelas em que a doença tem longa duração e os idosos. Assim, cabe aos médicos monitorar os indicadores do controle do diabetes: glicose em jejum, $\mathrm{HbA}_{1 \mathrm{c}}$, níveis de colesterol, pressão arteri$\mathrm{al}^{(58)}$ e creatinina. Resultados obtidos junto à população brasileira mostraram que exames como hemoglobina glicosilada e níveis de colesterol são escassamente registrados nos prontuários da rede pública ${ }^{(65)}$, o que pode retardar a identificação precoce de complicações crônicas microvasculares e macrovasculares.

Portanto, a avaliação da sensibilidade periférica deve fazer parte da rotina do exame físico das pessoas com diabetes, oportunidade em que o profissional deve descalçar o paciente para examinar seus pés à procura de sinais e sintomas precoces, por ser esta uma estratégia fundamental, embora ainda ela não faça parte da rotina da maioria dos serviços de saúde, apesar das recomendações internacionais ${ }^{(43,65)}$.

A equipe deve ser capaz de identificar, na sua atuação junto aos pa- cientes, as anormalidades precoces para lhes proporcionar educação contínua e lhes oferecer apoio na prevenção de úlceras e infecção de membros inferiores, mediante a categoria de risco identificado ${ }^{(58)}$.

O processo de educação fornecido à pessoa com diabetes deve ser reforçado a cada contato, de acordo com as necessidades relatadas e identificadas $^{(66)}$. Caberá ao profissional enfatizar os cuidados que o paciente deverá dispensar a seus pés no contexto de vida individual. Pessoas com baixo risco devem receber apoio para melhorar o controle glicêmico, níveis de lipídeos e pressão arterial, entretanto, aqueles com elevado risco precisarão adquirir habilidades para inspecionar seus pés, cuidar das unhas, selecionar os sapatos e, na ocorrência de algum ferimento, saber a quem recorrer ${ }^{(58)}$.

Nesta revisão foram descritas medidas básicas de avaliação passíveis de serem implementadas nas unidades de nível primário, com o intuito de rastrear o aparecimento de alterações em membros inferiores das pessoas com diabetes. Para tanto, considera-se importante que esses profissionais sejam sensibilizados para o risco de o paciente desenvolver pé diabético, por meio de cursos de capacitação e atualização profissional $^{(11)}$. Esse processo de sensibilização e capacitação deve ser contínuo, com avaliação periódica e discussão de casos clínicos, disponibilizando a eles instrumentos apropriados para esse fim.

Face à organização atual do sistema de saúde, o enfermeiro com treinamento específico para cuidar dos pés de pessoas com diabetes é apontado como o profissional que deve assumir a organização e cuidado dessa clientela. Suas habilidades devem levá-lo a detectar alterações neurológicas e vasculares periféricas, dermatológicas, além de outros agravantes que podem precipitar processos ulcerativos. Cabe, ainda, a este profissional, planejar interven- 
ções individualizadas, bem como incluir pessoas com diabetes nas atividades educativas.

A consulta de enfermagem apresenta-se como um fator importante de proteção ao agravo das complicações nos membros inferiores, visto que contribui para a forma de cuidar e educar, motivando o outro a participar ativamente do tratamento e a realizar o autocontrole, reforçando assim, sua adesão ao tratamento clínico ${ }^{(65)}$.

Diante do exposto, destaca-se a importância do atendimento primário no setor saúde, por meio da ampliação das ações básicas direcionadas aos cuidados com o diabetes e, particularmente, à prevenção de lesões nos membros inferiores resultantes do mau controle da doença e de práticas inadequadas aplicadas aos pés e unhas. Quanto às intervenções de baixa complexidade, estas podem e devem contribuir, decisivamente, para a prevenção de úlceras, minimizando a influência dos riscos, bem como o número de amputações ${ }^{(66)}$.

\section{REFERÊNCIAS}

1. Pedrosa HC, Nery ES, Sena FV, Novaes C, Feldkircher TC, Dias MSO et al. O desafio do projeto salvando o pé diabético. Terapia em Diabetes 1998; 4(19): $1-10$.

2. Bild DE, Selby JV, Sinnock P, Browner WS, Braveman P, Showstack JA. Lower-extremity amputation in people with diabetes epidemiology and prevention. Diabetes Care 1989; 12(1):24-31.

3. Caputo GM, Cavanagh PR, Ulbrecht JS, Gibbons GW, Karchmer AW. Assessment and management of foot disease in patients with diabetes. New Eng J Med 1994; 331(13): 856-9.

4. Levin ME. Foot lesions in patients with diabetes mellitus. Endocrinol Metab Clin North Am 1996; 25(2): 447-62.
5. Pecoraro RE, Reiber GE, Burgués EM. Pathways to diabetic limb amputation. Diabetes Care 1990; 13(5):513-21.

6. Reiber GE. Epidemiologia das úlceras e amputações do pé diabético. In. Bowker JO, Pfeifer MA. Levin e O’Neal o pé diabético. 6.ed, Rio de Janeiro: DiLivros; 2002. cap.2, p.13-33.

7. Most RS, Sinnock P. The epidemiology of lower extremity amputations in diabetic individuals. Diabetes Care 1983; 6:87-91.

8. Reiber GE. The epidemiology of diabetic foot problems. Diabet Med 1996; 13(Suppl 1):6-11.

9. Faglia E, Favales F, Morabito A. New ulceration, new major amputation, and survival rates in diabetic subjects hospitalized for foot ulceration from 1990 to 1993. Diabetes Care 2001; 24(1):78-83.

10. Spichler ERS, Miranda Jr F, Spichler ES, Franco LJ. Amputações maiores de membros inferiores por doença arterial periférica e diabetes melito no município do Rio de Janeiro. J Vasc Bras 2004; 3(2):111-22.

11. Gross JL. Detecção e tratamento das complicações crônicas do diabete melito Consenso Brasileiro. Arq Bras End Metab 1999; 43(1):7-13.

12. Reiber GE, Boyko EJ, Smirh DG. Lower extremity foot ulcers and amputations in diabetes. [online]. Disponível em: <http//:www.diabetes resource.com/html/edu10.htm>. (30 abr. 2001)

13. Sumpio B. Foot ulcers. New Engl J Med 2000; 343(11):787-93.

14. Boulton AJM. The diabetic foot: from art to science. The $18^{\text {th }}$ Camillo Golgi lecture. Diabetologia 2004. [on-line]. Disponível em: <http://www. springer link.com/media/...>. (4 ago. 2004)

15. Ramsey SD, Newton K, Blough D, McCulloch DK, Sandhu N, Reiber GE et al. Incidence, outcomes, and cost of foot ulcers in patients with diabetes. Diabetes Care 1999; 22(3):382-7.
16. Boyko EJ, Ahroni JH, Smith DG, Davignon D.Increased mortality associated with diabetes foot ulcer. Diabet Med 1996; 13:967-72.

17. Harrintong C, Zagari MJ, Corea J, Klitenic J. A cost analysis of diabetic lower extremity ulcers. Diabetes Care 2000; 23(9):1333-8.

18. Reiber GE, Lipsky BA, Gibbons GW. The burden of diabetic foot ulcers. Am J Surg 1998; 176(Suppl.2A):5-10.

19. Boike AM, Hall JO. A practical guide for examining and treating the diabetic foot. Cleve Clin J Med 2002; 69(4): 342-8.

20. Levin ME. Patogenia e tratamento geral das lesões do pé em pacientes diabéticos. In: Bowker JO, Pfeifer MA. Levin e O’Neal o pé diabético. 6a ed. Rio de Janeiro: Di-Livros; 2002. cap. 9, p. 221-61.

21. Fritschi C. Preventive care of the diabetic foot. Nurs Clin North Am 2001; 36(2):303-20.

22. Amadio AC, Sacco CN. Considerações metodológicas de biomecânica para a avaliação da distribuição da pressão plantar. Diabetes Clín 1999; 3(1):42-9.

23. Lavery LA, Armstrong DG, Vela SA, Quebedeaux TL, Fleischli JG. Practical criteria for screening patient at high risk for diabetic foot ulceration. Arch Int Med 1998; 158:157-62.

24. Porto CC. Exame clínico. $4^{\mathrm{a}}$ ed. Rio de Janeiro: Guanabara Koogan; 2000.

25. American Diabetes Association. Preventive foot care in people with diabetes. Diabetes Care 2001; 24 (Suppl 1):56-S7.

26. Boulton AJM. Lowering the risk of neuropathy, foot ulcers and amputations. Diabet Med 1998; 15 (Suppl 4):57-9.

27. Gamba MA. A importância da assistência de enfermagem na prevenção, controle e avaliação a pacientes portadores de diabetes com neuropatia e vasculopatia. Acta Paul Enferm 1991; 4(2/4):713. 
28. Grupo de Trabalho Internacional sobre Pe Diabético. Consenso internacional sobre pé diabético. Brasília: Secretaria de Estado de Saúde do Distrito Federal; 2001.

29. Mason J, O’Keeffet C, Mclntosh A, Hutchinson A, Booth A, Young RJ. A systematic review of foot ulcer in patient with type 2 diabetes. 1: prevention. Diabet Med 1999; 16: 801-12.

30. Mayfield JA, Reiber GE, Sanders LJ, Janisse D, Pogach LM. Preventive foot care in people with diabetes. Diabetes Care 1998; 21(12):2161-77.

31. Spollett GR. Preventing amputations in the diabetic population. Nurs Clin North Am 1998; 33(4):629-41.

32. Dahmen R, Haspels R, Koomen B, Hoeksma AF. Therapeutic footwear for the neuropathic foot. Diabetes Care 2001; 24(4):705-9.

33. Kumar S, Ashe HA, Parnell LN, Fernando DJS, Tsigos C, Young RJ et al. The prevalence of foot ulceration and its correlates in type 2 diabetic patients: a population-based study. Diabet Med 1994; 11:480-4.

34. Lavery LA, Vela SA, Fleischli JG, Armstrong DG, Lavery DC. Reducing plantar pressure in the neuropathic foot: a comparison of footwear. Diabetes Care 1997; 20(11):1706-9.

35. Pham H, Armstrong DG, Harvey C, Harkles LB, Giurini JM, Veves A. Screening techniques to identify people at high risk for diabetic foot ulceration: a prospective multicenter trial. Diabetes Care 2000; 23(5):606-11.

36. Sosenko JM, Sparling YH, Hu D, Welty T, Howard BV, Lee E et al. Comparison of quantitative sensory-threshold measures for their association with foot ulceration in the diabetic patients. Diabetes Care 1990; 13(10):1057-61.

37. Young MG, Bredy JL, Veves A, Boulton AJM. The prediction of diabetic neuropathic foot ulceration using vibration perception thresholds. Diabetes Care 1994; 17(6):557-60.
38. Abbott CA, Vileikyte L, Williamson S, Carrington AL, Boulton AJM. Multicenter study of the incidence of and predictive risk factors for diabetic neuropathic foot ulceration. Diabetes Care 1998; 21(7):1071-5.

39. McNeely MJ, Boyko EJ, Ahroni JH, Stensel VL, Reiber GE, Smith DG, Percoraro RE. The independent contributions of diabetic neuropathy and vasculopathy in the foot ulceration. Diabetes Care 1995; 18(2):216-19.

40. Armstrong DG. Loss of protective sensation: a practical evidence-based definition. J Foot Ankle Surg 1999; 38(1):79-80.

41. Collier JH, Brodbeck C. Assessing the diabetic foot: plantar callus and pressure sensation. Diabetes Educ 1993; 19(6):503-8.

42. Frykberg RG, Lavery LA, Pham H, Harvey C, Harkless L, Veves A. Role of neuropathy and high foot pressures in diabetic foot ulceration. Diabetes Care 1998; 21(10):1714-9.

43. Hutchinson A, Mclntosh A, Feder G, Home PD, Young R. Clinical Guidelines and Evidence Review for type 2 diabetes: prevention and management of foot problems. [online]. London: Royal College of General Practitioners, 2003. Disponível em: <http://www.rcgp.org.uk/ rcgp/clinspec/guidelines/diabetes $>$. (21 mar.2003).

44. Grupo de Trabalho Internacional sobre Pe Diabético. Diretrizes práticas: abordagem e prevenção de pé diabético. Brasília: Secretaria de Estado de Saúde do Distrito Federal; 2001.

45. Tanenberg RJ, Schumer MP, Greene DA, Pfeifer MA. Problemas neuropáticos das extremidades inferiores dos pacientes diabéticos. In. Bowker JO, Pfeifer MA. Levin e O’Neal o pé diabético. $6^{\mathrm{a}}$ ed. Rio de Janeiro: DiLivros; 2002. cap. 3, p. 34-65.
46. Cavanagh PR, Ulbrecht JS, Caputo GM. Biomecânica do pé no diabetes melito. In: Bowker JO, Pfeifer MA. Levin e O’Neal o pé diabético. $6^{\mathrm{a}}$ ed. Rio de Janeiro: Di-Livros. 2002. cap. 6, p. 125-95.

47. Ahroni JH, Boyko EJ, Forsberg RC. Clinical correlates of plantar pressure among diabetic veterans. Diabetes Care 1999; 22(6):965-72.

48. Veves A, Murray MJ, Boulton AJM. The risk of foot ulceration in diabetic patients with high foot pressure: a prospective study. Diabetologia 1992; 35:660-3.

49. Murray HJ, Young MJ, Hollis S, Boulton AJM. The association between callus formation, high pressures and neuropathy in diabetic foot ulceration. Diabet Med 1996; 13:879-82.

50. Pitel DL, Lord M, Foster A, Wilson S, Watkins PJ, Edmonds ME. Plantar pressures are elevated in the neuroischemic and the neuropathic diabetic foot. Diabetes Care 1999; 22(12): 1966-70.

51. Birke JA, Franks D, Foto JG. First ray joint limitation, pressure, and ulcera-tion of the first metatarsal head in diabetes mellitus. Foot Ankle Int 1995; 16(5):277-84.

52. Fernando DJS, Masson EA, Veves A, Boulton AJM. Relationship of limited joint mobility to abnormal foot pressures and diabetic foot ulceration. Diabetes Care 1991; 14(1):8-11.

53. Donatelli R. Normal biomechanics of the foot and ankle. J Orthop Sports Phys Therapy 1985; 7(3):91-5.

54. Simoneau GG, Ulbrecht JS, Derr JA, Becker MB, Cavanagh PR. Postural instability in patients with diabetic sensory neuropathy. Diabetes Care 1994; 17(12):1411-21.

55. Uccioli L, Faglia E, Monticone G Favales F, Durola L, Aldeghi A et al. Manufactured shoes in the prevention of diabetic foot ulcers. Diabetes Care 1995; 18(10):1376-8. 
56. Reiber GE, Smith DG, Wallace C, Sullivan K, Hayes S, Vath C et al. Effect of therapeutic footwear on foot reulceration in patients with diabetes. JAMA 2002; 287(19):2552-8.

57. Coleman W. Calçados para a prevenção de traumatismos: correlação com a categoria de risco. In: Bowker JO, Pfeifer MA. Levin e O’Neal o pé diabético. 6a ed. Rio de Janeiro: DiLivros; 2002. cap. 20, p. 620-34.

58. Rith-Najarian SJ, Reiber GE. Prevention of foot problems in persons with diabetes. J Fam Pract 2000; 49(Suppl 11):30-9.

59. Dargis V, Pantelejeva O, Jonushaite A, Vileikyte L, Boulton AJM. Benefits of a multidisciplinary approach in the management of recurrent diabetic foot ulceration in Lithuania. Diabetes Care 1999; 22(9):1428-31.
60. Calle-Pascual AL, Durán A, Benedi A, Calvo MI, Charro A, Diaz JA et al. A preventive foot care programme for people with diabetes with different stages of neuropathy. Diabetes Res Clin Pract 2002; 57:111-17.

61. Global Resource Center. Action plan for persons with diabetes. [online]. Disponível em: <http://www.diabetes resource.com/html/footcare $>$. (20 abr. 2001).

62. O'Connor PJ, Spann SJ, Wolf SH. Care of adults with type 2 diabetes mellitus: a review of the evidence. J Fam Pract 1998; 47(Suppl 5):13-22.

63. Lehto S, Rönnemaa T, Pyörälä K, Laakso M. Risk factors predicting lower extremity amputation in patients with NIDDM. Diabetes Care 1996; 19(6):607-12.
64. Clark Jr CM, Snyder JW, Meek RL, Stutz LS, Parkin CG. The national diabetes education program, changing the way diabetes: comprehensive diabetes care. Diabetes Care 2001; 24(4):617-8.

65. Gamba MA, Gotlieb SLD, bergamaschi DP, Vianna LAC. Amputações de extremidades inferiores por diabetes mellitus: estudo caso-controle. Rev Saúde Publica 2004; 38(3):399-404.

66. Pace AE, Foss MC, Ochoa-Vigo K, Hayashida M. Fatores de risco para complicações em extremidades inferiores de pessoas com diabetes mellitus. Rev Bras Enferm 2002; 55(5):514-21.
Ochoa-Vigo K, Pace AE. [Diabetic foot: strategies for prevention.] Acta Paul Enferm 2005; 18(1):100-9.

ABSTRACT: Approximately $50 \%$ of non-traumatic amputations of the lower limbs are realized among diabetes mellitus patients. Amputations are preceded by ulcers, characterized by skin erosion and loss of the epithelium, which can expand to the dermis or reach profound tissues, accompanied by insensibility due to chronic peripheral neuropathy, associated with small traumas as a result of inappropriate footwear, common dermatoses or incorrect foot manipulation. This study aims to describe the main findings and strategies to evaluate risk factors that lead to the development of lower limb complications among diabetes patients and make them part of the primary health care team's responsibilities. This literature review emphasizes evaluation of the feet and visible skin parts as a basic prevention measure, with a view to the early identification of neuropathic and peripheral vascular changes and biomechanical dysfunctions. The evaluation of footwear characteristics is highlighted as an external factor.

Descriptors: Diabetes mellitus; Risk factors; Diabetic foot.
Ochoa-Vigo K, Pace AE. [Pié diabético: estrategias para prevención.] Acta Paul Enferm 2005; 18(1):100-9.

RESUMEN: Aproximadamente $50 \%$ de las amputaciones no traumáticas en miembros inferiores ocurren entre personas con diabetes. Las amputaciones son antecedidas por úlceras, caracterizadas por lesiones cutáneas con pérdida del epitelio, que pueden extenderse a la dermis y llegar hasta tejidos profundos. Las úlceras, generalmente, son acompañadas de insensibilidad por neuropatía periférica crónica e asociadas a pequeños traumas, originadas por uso de calzados inapropiados, dermatosis común o manipulaciones incorrectas de los pies. El objetivo del estudio es describir las principales estrategias para evaluar los factores de riesgo que conducen al desarrollo de complicaciones en miembros inferiores distales entre personas con diabetes, bien como considerarlos una responsabilidad del equipo de asistencia primaria. Es un trabajo de revisión de la literatura, con énfasis en la realización del examen físico de los pies, como medida preventiva fundamental, para identificar, precozmente, alteraciones neurológica y vascular periféricas y disfunciones biomecánicas. Destacase el examen minucioso de los calzados como factor externo.

Descriptores: Diabetes mellitus; Factores de riesgo; Pié diabético 